\title{
Pressure wave propagation in a shaken granular bed
}

\author{
Stephen R. Hostler* and Christopher E. Brennen \\ California Institute of Technology, Pasadena, California 91125, USA
}

(Received 15 March 2005; revised manuscript received 29 July 2005; published 21 September 2005)

\begin{abstract}
Pressure waves in a granular material travel through particle contact points and are primarily transmitted by the "force chains" that carry most of the load in a granular medium. However, these force chains tend to be fragile and ephemeral and can be disrupted by very minor perturbations including the waves themselves. External vibration also disrupts the force chains and therefore also changes the wave propagation characteristics. In this paper we study the effects of vibration on wave propagation in a shaken granular bed.
\end{abstract}

DOI: 10.1103/PhysRevE.72.031304

PACS number(s): 45.70. $-\mathrm{n}, 62.30 .+\mathrm{d}$

\section{INTRODUCTION}

The first paper in this series [1] described experiments and the corresponding computer simulations for wave propagation in a static granular bed. These identified several features of the wave propagation characteristics that were associated with the process of propagation along dominant force chains and, particularly, the complexity that arises because of the fragility of those force chains [2-6]. It is this heterogeneous and ephemeral nature of a granular bed that leads to complicated and often elusive wave propagation characteristics. In the present paper, the investigation is pursued further by shaking the entire bed and therefore altering the force chain microstructure within the granular bed.

Though there have been a number of previous experimental investigations of wave propagation in granular beds [7-11] (for a review, see Ref. [1]), this is the first attempt to perform measurements in a bed subject to agitation. Of course, a fluidized bed is highly agitated and so one possibility would be to find linkage to the experiments measuring wave propagation in fluidized beds [12-14].

The present experiments and simulations first measure the wave speed and the attenuation in the agitated granular bed. Details concerning the experimental setup and the configuration of the simulations can be found in part I of this series. With respect to attenuation we note that there are a number of mechanisms through which wave energy is dissipated in a granular bed. Since particle contacts are inelastic and frictional, some energy is lost during particle interactions. Frictional effects convert some of the wave energy into heat. Plastic deformation of the particles can also absorb some of the wave energy. Other losses can be associated with particle rearrangement within the granular bed and/or the scattering of wave energy through the particle contact network. The body of work on dissipation of waves in granular materials is very limited.

\section{CONTINUOUS WAVES IN AN AGITATED GRANULAR BED}

First we examine the agitated granular beds with a sinusoidal wave input. Figure 1 presents typical raw signals from

\footnotetext{
*Currently at the Department of Mechanical and Aerospace Engineering, Case Western Reserve University. Electronic address: hostler@case.edu
}

an experiment with imposed vertical shaking at $20 \mathrm{~Hz}$ $\left(f^{*}=1.13 \times 10^{-5}\right)$. Note that all three sensors not only detect the low-frequency agitation introduced by shaking, but also record the horizontal input waves. This dispelled initial doubts that the higher-frequency waves could be detected in the presence of shaking. It transpired that the higherfrequency waves were readily detectable up to shaking levels of the order of $2 g$. Note also that the sound amplitude is smallest when the state of the bed is most compressed. This occurs because compression restricts the motion of the piston and thus causes a smaller acceleration amplitude.

Typical spectra of these signals are presented in the upper right plot of Fig. 2. Peaks in the pressure transducer spectra occur at the input wave frequency $(600 \mathrm{~Hz})$ and the agitation frequency $(20 \mathrm{~Hz})$. In addition, there are sidebands at $20-\mathrm{Hz}$ intervals around each of these peaks. The sidebands on both sides of the input wave frequency are indicative of some quadratic nonlinearity. As evidenced by the relatively small peak in its spectrum at $20 \mathrm{~Hz}$, the piston accelerometer is much less sensitive to the shaking than either transducer.

Looking at the data in more detail, Fig. 2 shows the evolution of the spectra as the shaking amplitude is increased. Without shaking, an input wave at $600 \mathrm{~Hz}$ also exhibits a second harmonic at twice this frequency. The introduction of agitation leads to a peak at the agitation frequency $(20 \mathrm{~Hz})$ and to the sidebands mentioned previously. Sidebands are also present on the harmonic at $1200 \mathrm{~Hz}$. As the shaking amplitude is increased further, the harmonic of the input wave source is reduced in favor of more sidebands around

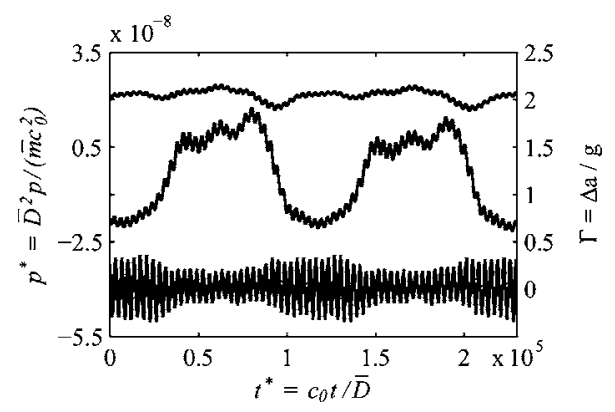

FIG. 1. Raw signals for the piston accelerometer, near pressure transducer, and far pressure transducer (bottom, middle, and top, respectively) for a vertically agitated bed with sound input in a horizontal direction. 

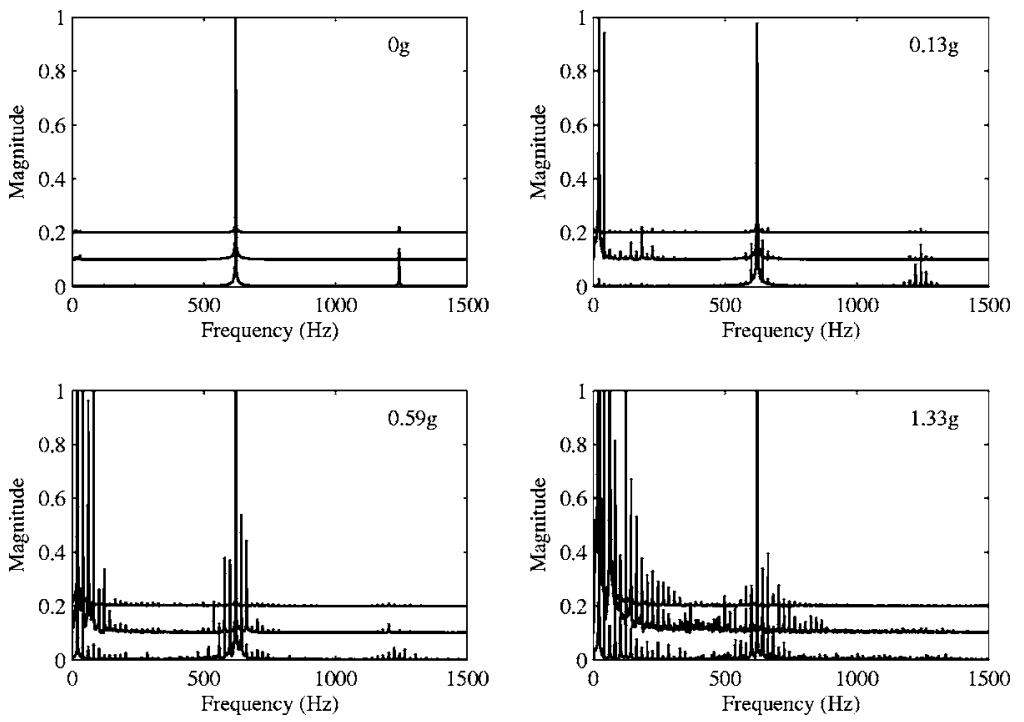

FIG. 2. Spectra for the accelerometer (bottom), near pressure transducer (middle), and far pressure transducer (top) for an increasingly agitated bed with sound input in a horizontal direction. The shaking acceleration amplitude is included in each plot. both the shaking frequency and the input wave frequency. For the largest amplitudes of shaking, the sidebands span the entire frequency range between the input wave frequency and the agitation frequency.

Measurements of the wave speed in the presence of agitation are shown in Fig. 3. It appears that agitation leads to a change of state of the bed. The constant reordering of force chains brought on by the shaking constantly shifts the paths of wave propagation which leads to changing wave speed. Thus, at low levels of shaking there is a large difference between the measured wave speed depending on the prior history of shaking of the bed. For both the glass and PVC particles, there is as much a $50-\mathrm{m} / \mathrm{s}$ difference in the wave speed at the same level of shaking. In this regime, wave propagation is governed by the geometric configuration of the bed which is constantly changing as a result of the imposed shaking. With larger shaking amplitude, a transition is observed and the variability of the wave speed measurements is significantly reduced. This transition occurs at somewhat different levels of shaking for the glass $(0.5 \mathrm{~g})$ and PVC particles $(0.8 g)$, but both levels are below the acceleration at which the particles detach from the base of the container $(1 g)$. Beyond this transition point, the wave speed is single valued, suggesting that the state of the bed is now determined by the current level of agitation rather than any past history. Moreover, at all levels of shaking, the scatter in the wave speed measurement is significantly smaller for the PVC particles than for the glass particles. This difference may be due to the fact that the regular, spherical glass particles are more likely to undergo rearrangement than the cylindrical PVC particles. This could explain both the reduced scatter and the higher transition level for the PVC particles.

\section{SIMULATIONS OF CONTINUOUS WAVES}

Simulations were carried out with the same excitation levels as used in the experiments and the overall results were similar. After just two or three shaking cycles, the pressure signals at both the left and right walls of the simulation cell settle into the typical pattern shown in Fig. 4 (top). In addi- tion to the signal envelopes corresponding to the shaking frequency, the signals clearly contain the high-frequency wave input. This is qualitatively similar to the experimental measurements shown in Fig. 1. Since the high-frequency signal in Fig. 4 (top) is so cleanly superimposed on the shaking frequency, cross correlation is readily accomplished and was used to obtain a wave speed.

Another interesting feature of Fig. 4 (top) is the timevarying amplitude of the high-frequency waves. The amplitude at the peak of the shaking envelope when the bed is in a more compressed state is significantly larger than that in the trough. This is a consequence of the nonlinearity of the contacts, the same displacement producing a greater force (pressure) in a chain when the mean level of the pressure is higher.

Obviously, as the shaking level is increased the particle contacts become more and more ephemeral and the mechanisms of wave propagation change. Above some level of shaking particle chains cannot persist and the granular bed changes to a highly "thermalized" state in which waves will only propagate at the typical velocity of particles in between

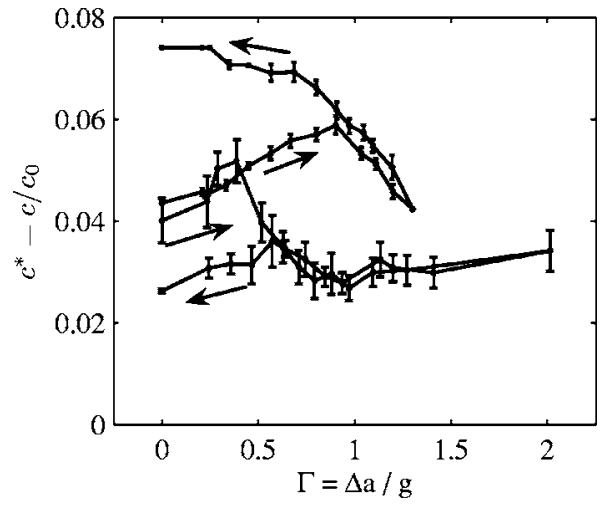

FIG. 3. Phase speed in a shaken bed as the shaking amplitude is first increased and then decreased (shown by arrows). Each data point is an average of 13 samples; error bars show the standard deviation. Measurements with PVC particles (upper curve) and 4-mm glass spheres (lower curve). 

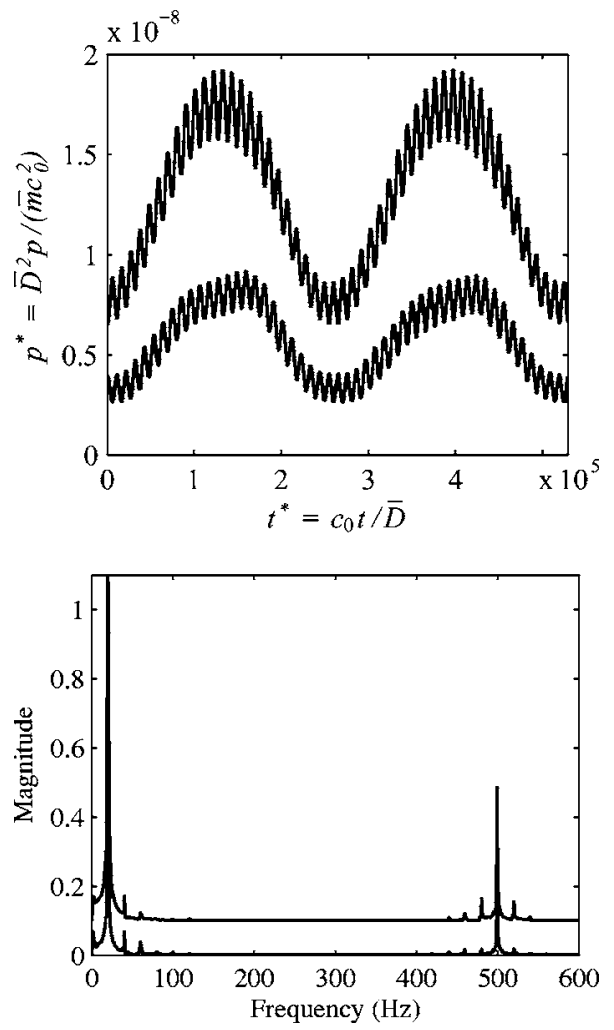

FIG. 4. Top: typical pressure signals at the left (input) wall (upper curve) and at the right wall (lower curve) at the half depth of the simulation cell for combined vertical shaking $(20 \mathrm{~Hz}, 0.5 \mathrm{~g})$ and horizontal wave input $(500 \mathrm{~Hz}, 0.2 \mathrm{~g})$. Bottom: typical spectra of the pressure offset vertically for clarity in the same order as the pressure signals.

collisions. However, in the present experiment, that granular state changes throughout the agitation cycle, and there are both compressed and rarefied zones within the bed. Wave propagation may still occur in the compressed zones. Examples of the pressure recorded at the wall of the simulation cell during one period of large amplitude shaking are shown in Fig. 5. Note the intermittent wave propagation; the wave is evident during only a part of the agitation period-from $t^{*}=1.5 \times 10^{4}$ to $3 \times 10^{4}$ in this example. Over the rest of the cycle, the pressure is either zero or large and very noisy. Regions of zero pressure indicate that the particles are out of contact with the sensor. The large pressure spike near the beginning of the trace is the result of the impact of the granular bed with the base of the simulation cell. That impact and the noise it generates obscure the basic signal.

Wave speeds in the simulations were assessed for a range of agitation amplitudes by evaluating the phase shift in the recorded pressure over an entire period of the agitation cycle; thus they represented an average over a range of compressed and rarefied states. The speeds thus obtained are shown in Fig. 6 and exhibit some of the same behavior seen in the experiments. The wave speed takes different values depending on the prior history of shaking but to a smaller degree than in the experiments. This wave speed variation was observed to increase as the size of simulated sensor was reduced relative to the particle diameter. Unlike the experi-

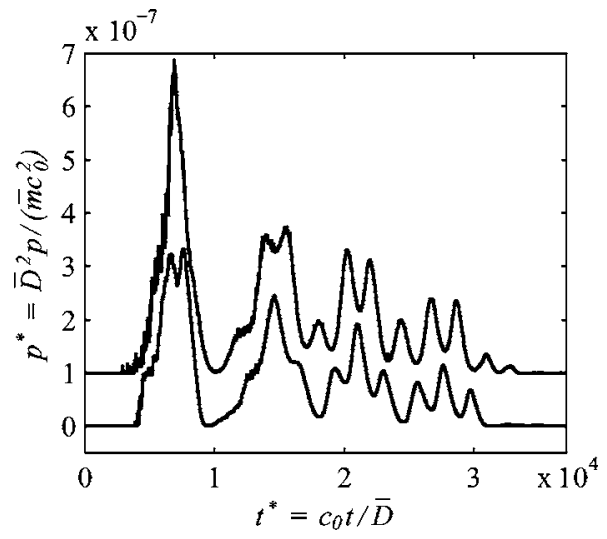

FIG. 5. Typical pressure signals during large amplitude excitation: at the left (input) wall (upper curve) and at the right wall (lower curve) at the half depth of the simulation cell for combined vertical shaking $(20 \mathrm{~Hz}, 1.25 \mathrm{~g})$ and horizontal wave input $(500 \mathrm{~Hz}$, $1.0 \mathrm{~g}$ ). The upper curve is offset vertically for clarity.

ments, there is no observed shaking threshold in the simulations beyond which the wave speed is single valued.

\section{EXPERIMENTS AND SIMULATIONS USING SINGLE INPUT PULSES}

Experiments and simulations were also conducted to examine the propagation of single pulses in the shaken bed. Since the pulse widths are much shorter than the agitation period, one is essentially examining the wave propagation characteristics of the bed at a particular instant during the agitation cycle. At that particular instant, one part of the bed may be in a greater state of compression than another and the wave propagation patterns are therefore more complex than in a static bed. Typical pressures caused by the shaking alone are seen on the left-hand side of the upper graph in Fig. 7. The pressure on the more distant transducer is quite sinusoidal. However, for some unknown reason, there is a frequency-doubling feature in the signal from the closer transducer. Note that on both transducers the shaking signal is remarkably consistent from one period to the next. This

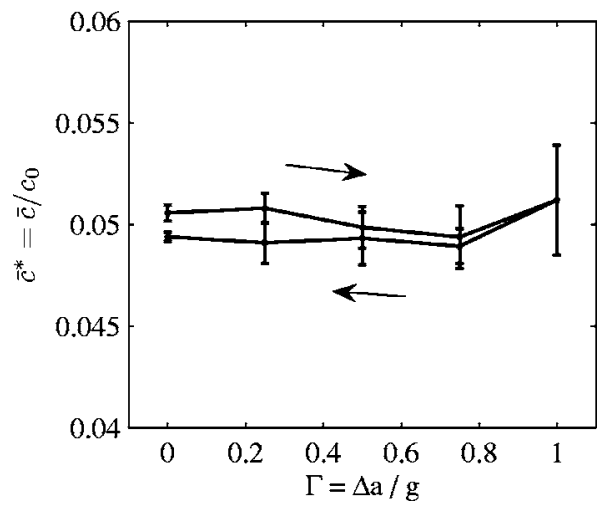

FIG. 6. Phase speed in a simulated granular bed as the shaking amplitude is first increased and then decreased (shown by arrows). Each data point is the average of nine sequential measurements; error bars show the standard deviation. 

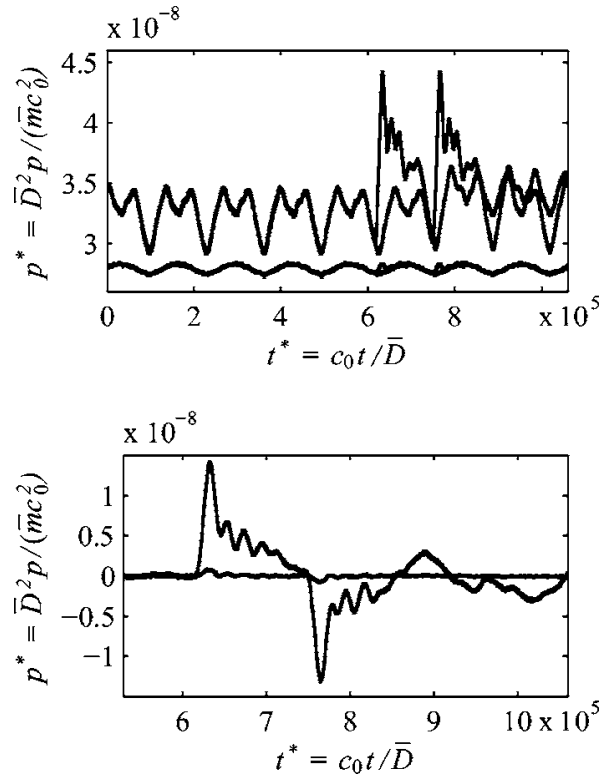

FIG. 7. Top: signals from the near (upper) and far (lower) transducer combined with the signals shifted by one period of the shaking. Bottom: the expanded result of subtracting the shifted signal from the original signal.

repeatability allows for a unique bit of data analysis as follows. The input pulse from the piston produces the first or (left most) pulse in the signals from the transducers in the upper graph of Fig. 7. Overlaid on top of these records are the same signals digitally shifted in time by one shaking period. Subtracting these phase-shifted signals from the original signals produces the typical result shown in the lower graph. In this way the shaking component in the original signal is removed (the second inverted signal was simply ignored) and these stripped signals from the two transducers can then be compared to obtain the wave speed and the attenuation. Indeed, the stripped signals typified by the lower graph were similar to the signals recorded in the absence of shaking. Specifically they comprise a primary peak followed by a series of oscillations (in Ref. [1] we propose that these trailing oscillations may result from natural oscillation of the force chains). The primary peak pulses have many of the same characteristics of single pulses in static beds [1] including the propensity for semipermanent waves $[15,16]$ resulting from short input pulses.

We now shift attention to the computer simulations which have the advantage that the structure of the wave within the bulk of the granular bed can be more fully dissected. Sample results from the simulations are shown in Figs. 8 and 9. Note that the pulse width (shown by the interval on the extreme left of Fig. 9) and its propagation duration are very short compared with the period of the shaking so that the pulse essentially experiences a bed state that is frozen at a particular moment in the shaking period; thus the pulse propagation acts as a diagnostic of the state of the bed at that moment.

One example of the progress of a pulse wave as it propagates is shown in Fig. 8 where the particles have been grey scale coded to indicate the magnitude of the net horizontal force on each particle: black corresponds to zero force and
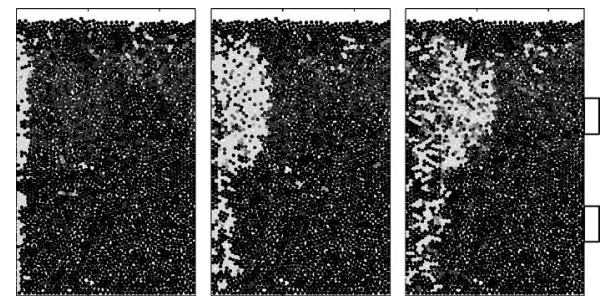

FIG. 8. Transmission of a pressure pulse in a shaken granular bed at the particular moment described in Fig. 9. Three instants are shown separated by intervals of $\Delta t^{*}=c_{0} \Delta t / \bar{D}=250$. The size and location of the pressure sensors used for Fig. 9 are shown in the third frame.

white to a dimensionless force of $F^{*}=\bar{D} F / \bar{m} c_{0}^{2}=5 \times 10^{-9}$. The particular moment in the shaking cycle at which this pulse was generated and propagated is shown by the inset in Fig. 9; it is just after the bed floor has passed through its maximum elevation and is being pulled away from the bed faster than the bed can fall. This has generated an expansion wave that has traveled upward from the floor and created, at this moment in time, a zone of low pressure that takes up the lower half of the depth of the bed. Many of the particles in this zone have little or no contact with surrounding particles. Consequently the progress of the pulse through this lower half of the bed is quite different from its transmission through the upper, compressed half of the bed. The left-hand frame of Fig. 8 shows the pulse just after its creation at the left wall. The middle frame shows that a fairly coherent wave is progressing through the compressed upper half of the bed while a much slower and less-well-defined wave is traveling through the lower half. The right-hand frame serves to demonstrate that the wave front is much better defined in the upper half than in the lower half.

Figure 9 shows the pressure recorded at the right wall of the simulation box on a 10-particle wide sensor that is placed at a depth 26 particles below the upper surface. This sensor is therefore in the upper half of the bed and consequently

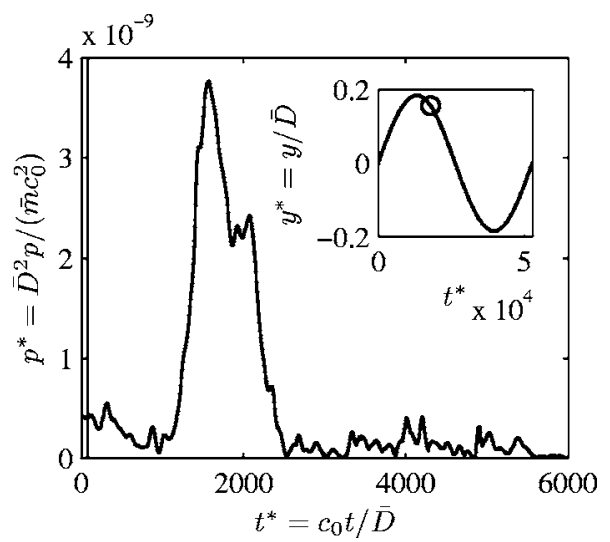

FIG. 9. Pulse recorded on the right wall of the simulation as a result of a narrow pulse applied to the left wall. Pressure is measured on a 10-particle-wide sensor on the right wall 26 particles below the upper surface. The base of the box is shaken with an amplitude of $1.5 \mathrm{~g}$. The inset shows the moment (circle) during the shaking cycle at which the pulse was generated and propagated. 


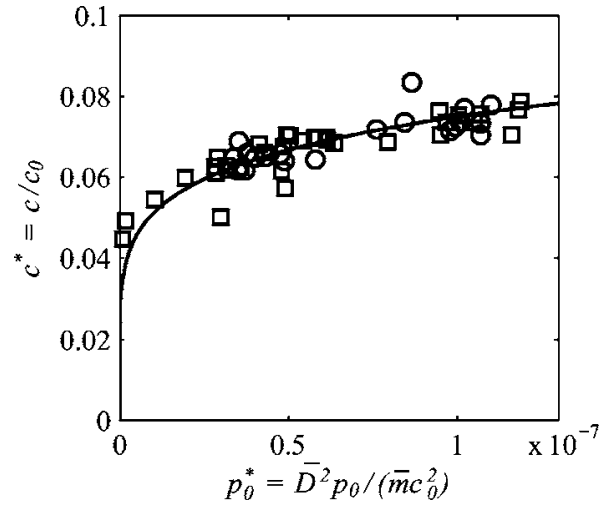

FIG. 10. Wave speed as a function of the confining pressure $p_{0}^{*}$ for pulsed wave simulations. Results from agitated ( $\square$ ) as well as unagitated $(\bigcirc)$ cases are plotted.

records the arrival of a pulse that is similar to those recorded in the absence of shaking [1]; there are, however, some notable differences which we detail below. First, however, it is important, to record that a similar sensor located at a depth 56 particles below the upper surface recorded no measurable signal on the scale of Fig. 9. In other words, the particle contacts are so few and so weak in the lower half of the bed that none of the pulse reaches the right wall.

Differences between the received pulse in the upper half as shown in Fig. 9 and the received signals in an identical static or nonshaken bed for the same input pulse were as follows. First the amplitude of the pulse shown in Fig. 9 is several orders of magnitude smaller than the received pulses in the static beds. This is the result of a much lower confining pressure compared to the nonshaken bed. In addition the pulse in Fig. 9 is noisier; the trailing edge of the wave has additional localized peaks that may be the result of more contorted force chains.

\section{PULSE WAVE SPEEDS}

The dependence of the wave speed on the local pressure is shown in Fig. 10 where results from the current shaking simulations and from the static bed simulations of Ref. [1] are both plotted. The current shaking simulations effectively extend the range of pressures for which simulations were made. In Fig. 10, the two sets of unagitated bed data correspond to wave speeds evaluated at two depths in a static bed. These are augmented by simulations at higher-pressure levels made in the current tests during the compressed phase of the agitation cycle and by simulations at lower pressures obtained during the expansion phase.

When plotted against the local confining pressure, the results from the unagitated and agitated bed cases coincide. The trend is an increase in the wave speed with increasing local confining pressure $p_{0}^{*}$. As shown in the figure, a 1/6 power law provides a good fit to the data and is therefore in good agreement with the Hertzian contact theory as described by Goddard [17]. Note that Goddard predicts a 1/4 power relation in real particle beds where the change from $1 / 6$ to $1 / 4$ is due to particle asperities. Since asperities are not included in the present simulations, the $1 / 6$ power dependence is expected to hold over the entire pressure range.

\section{CONCLUDING REMARKS}

Taken in conjunction with the earlier results for a static granular bed [1], the present experiments and simulations of wave propagation through agitated or shaken granular beds shed further light on the processes of wave propagation through the fragile and ephemeral force chain networks that carry elastic waves in granular material. In particular, lowfrequency shaking of the bed provides active, external disruption of the force chain network. At the outset, there were questions as to whether the high-frequency propagating waves and pulses could be discerned within the noise generated by the shaking, but aided by some special signal processing, it transpired that this was possible up to surprisingly high amplitudes of shaking (up to $2 g$ ). However, as the shaking amplitude was increased, beat frequencies that were observed at low amplitudes gradually evolved into a broad spectrum of noise.

The spectra of the signals in the sinusoidal input experiments demonstrated the nonlinear nature of wave propagation in an agitated granular bed. In addition to peaks from the input wave and agitation frequencies, beat frequencies were observed at intervals of the shaking frequency about the input wave frequency. The number of beat frequencies increased with the agitation acceleration amplitude. Such beating is indicative of a quadratic nonlinearity in the system. The corresponding simulations showed identical behavior in their spectra.

Wave speed measurements for the sinusoidal input experiments showed interesting behavior as the agitation amplitude was increased. For low shaking amplitudes, the wave speed took different values after undergoing an increasing then decreasing shaking cycle. Differences in the measured speed could be as great as $50 \mathrm{~m} / \mathrm{s}$. At sufficiently high shaking amplitudes the wave speed became single valued. In this range, it appeared that the wave speed was governed more by the granular state rather than the geometrical configuration of the bed. The transition to this single-valued regime occurred at a different agitation amplitude for the glass spheres than the PVC cylinders. Simulations failed to exhibit such a high degree of wave speed variation at low agitation levels, suggesting that the contact model used is not adequate in this respect. Other inadequacies of the contact model are discussed in Ref. 1.

Simulations with sinusoidal input did show the temporal variation of the granular bed state at various intervals during a shaking cycle. The input wave could only be detected during parts of the agitation period. At times when the bed was locally expanded, zero pressure was detected. During highpressure events, such as the collision of the bed floor with the granular bed, the input source was lost in this noisy pressure spike. The input wave signal was only evident at times when the bed was sufficiently compressed.

Simulations with a pulsed input further examined the effect of the state of the granular bed on wave propagation properties through it. Pressure traces and visualizations of 
forces within the bed showed that due to state changes induced by the shaking, wave propagation could be spatially localized. Propagation was possible in the upper layers of the bed where the bed was in a relatively compressed state. On the other hand, expanded regions in the bed precluded elastic wave propagation. In the region where propagation occurred, the wave speed was found to be lower than that measured in an unagitated bed. The slower wave was attributed to a lower local confining pressure due to agitation. Thus the parameter that most affects the wave speed is the local confining pressure. As observed by Potapov and Campbell [18], the solid fraction is a weak indicator of changes in the contact condition between particles. A significant change to the bed microstructure is required to significantly influence the solid fraction. In contrast, imperceptible changes in the microstructure were seen to affect wave propagation [2,3]. The local confining pressure is a much better predictor of such changes at the particle contacts.
[1] S. R. Hostler and C. E. Brennen, preceding paper, Phys. Rev. E 72, 031303 (2000).

[2] P. K. Haff (unpublished).

[3] C. Liu and S. R. Nagel, Phys. Rev. B 48, 15646 (1993).

[4] C. Liu and S. R. Nagel, J. Phys.: Condens. Matter 6, A433 (1994).

[5] C. H. Liu, Phys. Rev. B 50, 782 (1994).

[6] J. Geng, D. Howell, E. Longhi, R. Behringer, G. Reydellet, L. Vanel, E. Clément, and S. Luding, Phys. Rev. Lett. 87035506 (2001).

[7] J. Duffy and R. Mindlin, J. Appl. Mech. 24, 585 (1957).

[8] B. Hardin and F. Richart, J. Soil Mech. Found. Div. 89, 33 (1963).

[9] C. H. Liu and S. R. Nagel, Phys. Rev. Lett. 68, 2301 (1992).

[10] G. Ben-Dor, A. Britan, T. Elperin, O. Igra, and J. Jiang, Exp.
Fluids 22, 432 (1997).

[11] X. Jia, C. Caroli, and B. Velicky, Phys. Rev. Lett. 82, 1863 (1999).

[12] W. Gregor and Rumpf, Int. J. Multiphase Flow 1, 753 (1975).

[13] D. Musmarra, M. Poletto, S. Vaccaro, and R. Clift, Powder Technol. 82, 255 (1995).

[14] G. J. Weir, Chem. Eng. Sci. 56, 3699 (2001).

[15] V. F. Nesterenko, Dynamics of Heterogeneous Materials (Springer-Verlag, New York, 2001).

[16] S. Sen, M. Manciu, R. S. Sinkovits, and A. J. Hurd, Granular Matter 3, 33 (2001).

[17] J. Goddard, Proc. R. Soc. London, Ser. A 430, 105 (1990).

[18] A. V. Potapov and C. S. Campbell, Phys. Rev. Lett. 77, 4760 (1996). 